\title{
Pit Crew Approach to Pre Hospital Trauma Resuscitation
}

\author{
David Menzies, Ray Quinn, Angela Sheridan, Danny Dowdall, Mark O’Byrne, Mark O’Neill, Phil Darcy
}

Motorcycling Ireland Medical Team

\section{Introduction \& Aims}

Pre hospital trauma care is often delivered by dual crewed ambulances supported by additional resources as necessary and available. Coordinating resuscitation of a critically injured patient may require multiple simultaneous actions. Equally a large number of practitioners can hinder patient care if not coordinated. We aim to describe a multidisciplinary, scalable approach to pre hospital trauma care suitable for small and large multi disciplinary teams.

\section{Methods}

The $\mathrm{MCl}$ Medical Team (as part of Motorsport Rescue Services) is a PHECC-registered multidisciplinary team which provides medical cover at motorcycle road racing events in Ireland. The $\mathrm{MCl}$ Medical Team has significant experience of major trauma and routinely performs pre hospital anaesthesia for trauma patients.

We have evolved a pit crew approach to trauma care with predefined roles and interventions assigned to a five person team: three clinical members, a scribe and a team lead. The approach is both scalable and collapsible, meaning that if multiple patients are present, roles can be merged; if additional clinical input is required, roles can also be supplemented. Each team member carries equipment and medications specific to their role, allowing efficiencies at the patient's side.

\section{Principles}

- Simple, straightforward system

- Allows coordinated simultaneous activity

- All team members cross -trained insofar as possible.

- Roles pre-assigned.

- If less than 5 team members initially, assume in order of $A, B, C$ sequence.

- Clinical team members feed back to team leader.

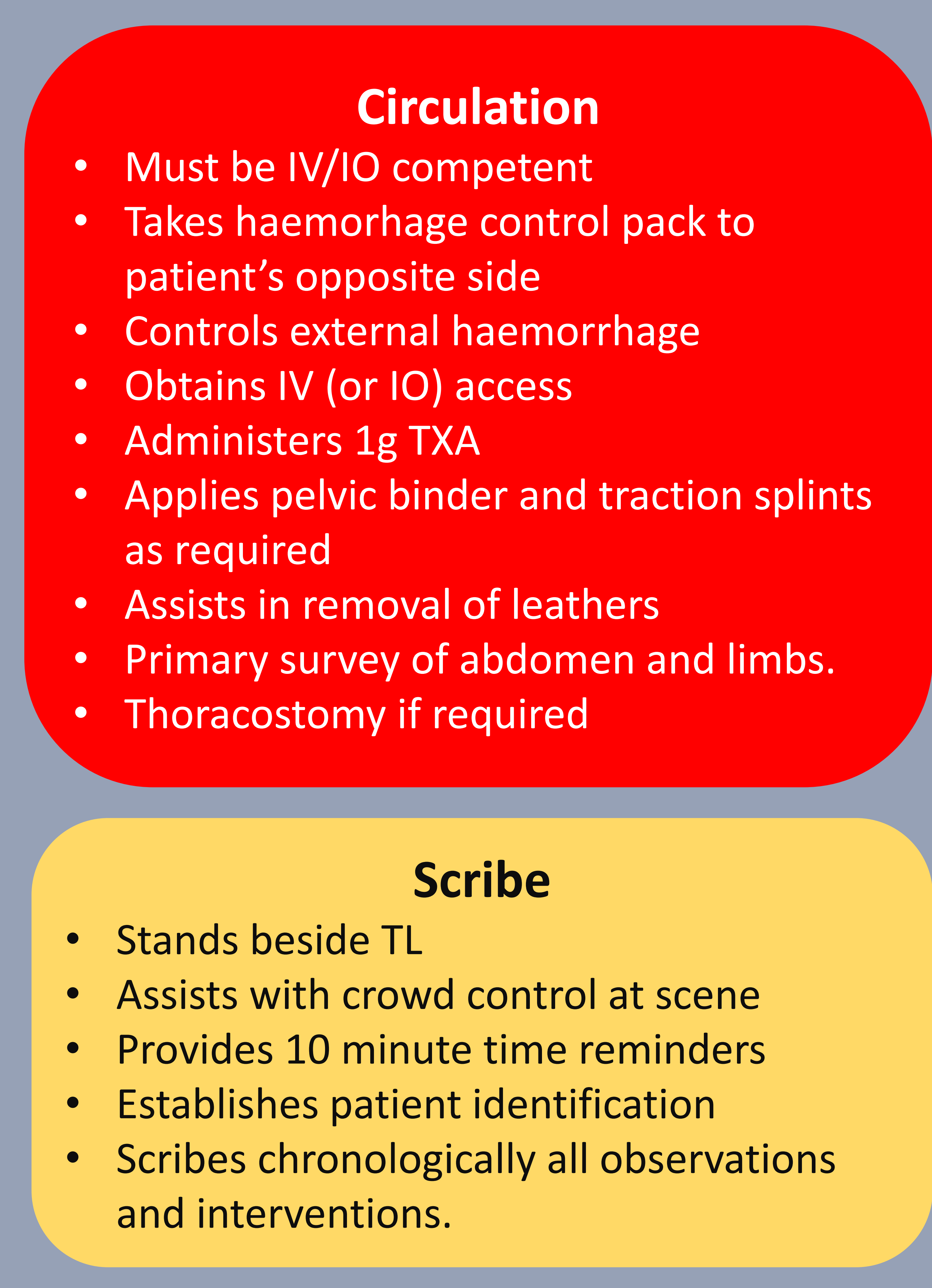

\section{Results \& Conclusions}

The pit crew approach to pre hospital trauma care has evolved over a decade and is routinely implements at motorcycle road races in Ireland. The pit crew trauma approach, although applicable to a pre defined five person team in unique circumstances, may also be applicable to ad hoc clinical teams that typically form in the pre hospital arena.

\section{Breathing}

Trained to RSI kit dump and assist

- Takes cardiac monitor to patient's most accessible side

- Assists with helmet removal

- Applies all monitoring

- Primary survey of breathing \& chest

- Thoracostomy if required

\section{Team Leader}

- Not necessarily clinical lead

- Stands back

- Brings trauma drugs bag to patient

- Directs primary survey

- Defines order of therapies according to clinical lead

- Directs additional resources

- Logistical planning and extrication

- Monitors scene time

\section{References}

1. PHECC Clinical Practice Guidelines, 2017

2. ATLS manual, 2016

3. ATACC manual, 2014

4. $\mathrm{MCl}$ Medical Team SOPs, 2018 